\title{
Design of a Leg-Wheel Hybrid Mobile Platform
}

\author{
Shuan-Yu Shen, Cheng-Hsin Li, Chih-Chung Cheng, Jau-Ching Lu, Shao-Fan Wang, and Pei-Chun Lin
}

\begin{abstract}
We introduce the design of a leg-wheel hybrid platform Quattroped. Comparing to most hybrid platforms which have separate mechanisms of wheels and legs, this robot is implemented with a transformation mechanism which directly changes the morphology of wheels (i.e. a full circle) into 2 degree-of-freedom legs (i.e. combining two half-circles as a leg). The mechatronics, software infrastructure, and the initial experimental test of the robot are also reported.
\end{abstract}

\section{INTRODUCTION}

Legs and wheels are two widely adopted methodologies utilized on the ground locomotion platforms. After a long evolution process, most ground animals are evolved with agile and robust legs which are capable of driving their body to move on the uneven natural terrains smoothly and rapidly. Wheels, in contrary, are the smart human inventions specialized in rolling on the flat ground, whose excellent performance of power efficiency and traveling speed sets a high standard which can hardly be competed by legs. Thus, a leg-wheel hybrid platform with great mobility on both flat grounds (by wheels) and rough terrains (by legs) seems to be on the track of "future" mobile platforms suitable for general indoor-outdoor environments.

The leg-wheel hybrid robots can generally be classified according to their morphology [1]. One of the popular category is "articulated-wheeled" robots, where the robots usually combine active or passive wheels on the feet of articulated legs. For example, Roller Walker [2] which incorporates a passive wheel on the foot of each 3 degree-of-freedom (DOF) leg, so the locomotion can be switched from quadrupedal walking into roller skating on the flat ground. Bipedal walker WS-2 has wheel driving mode when the wheeled foot module W-16 is mounted [3]. Stair-climbing robot Zero Carrier [4] has four active and four caster wheels on the feet of its eight unified prismatic joint legs, which improves the mobility on the flat ground. Similarly, robot PAW [5] adds four active wheels on the distal ends of its compliant legs of the original quadruped Scott II. Similar strategy is also adopted by robot Walk'n Roll [6]. Hylos [7] utilizes active 2-DOF suspension mechanisms on its four wheels to improve its mobility on the uneven terrain. The other popular category is "leg-wheel separated" robots, where the robots have both legs and wheels mounted on the

This work is supported by National Science Council (NSC), Taiwan, under contract NSC 97-2218-E-002-022 and by Ministry of Economic Affairs, Taiwan, under contract 97-EC-17-A-04-S1-054.

Authors are with Department of Mechanical Engineering, National Taiwan University, No.1 Roosevelt Rd. Sec.4, Taipei, Taiwan. Corresponding email: peichunlin@ntu.edu. tw body, and the motion of the robot is generated by collaboration of these two mechanisms. For example, Chariot III [8] has two big wheels and four 3-DOF legs. Wheeleg [9] has two pneumatically actuated 3-DOF front legs and two independently driven rear wheels. A conventional wheelchair with two front legs improves its mobility on step crossing [10]. Some other morphologies are also proposed by researchers. For example, Octal Wheel [11] has a special wheel-arm mechanisms which is comprised by an arm with two wheels mounted on each side, and the robot is capable of climbing over obstacles such as stairs. Whegs [12] driven by four 3spoke wheels without "rims" has excellent mobility on both flat and rough terrains. RHex [13], [14] with six 1 rotational DOF half-circle legs can simply generate various legged behaviors via open-loop control, and the robot can be transformed into a six-half-wheeled robot if the rotational axes are shifted. Some other robots using tracks as legs have been reported as well [15]-[17].

Here, we adopt a different methodology and design a leg-wheel hybrid robot shown in Figure 1 by incorporating a "transformation mechanism" which directly changes the morphology of wheels (i.e. a full circle) into legs (i.e. combining two half-circles as a leg). The actuators are carefully positioned so the robot can perform in both wheel mode (i.e. the wheel moves in 1-DOF rotational motion) and leg mode (i.e. the leg moves in 2-DOF planar motion) correctly.

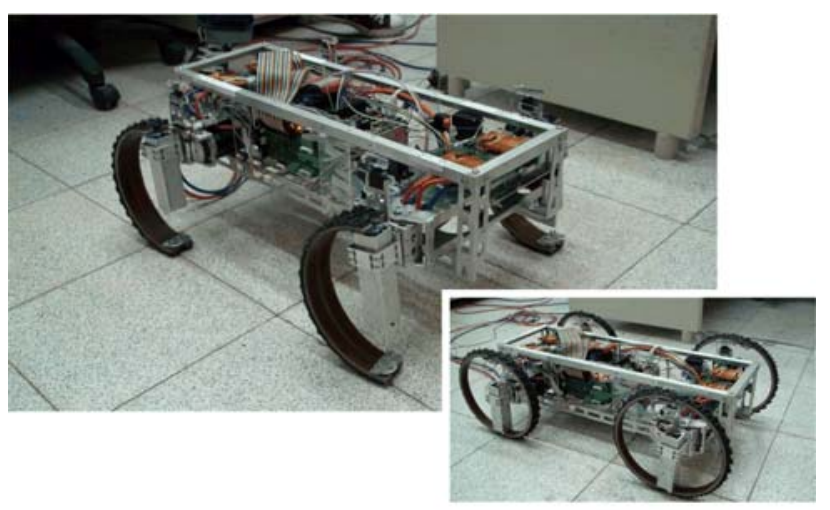

Fig. 1. Picture of Quattroped: a leg-wheel hybrid mobile platform.

Section II introduces the design concept of this robot, followed by Section III which describes the leg-wheel transformation mechanism in detail. Section IV reports the mechatronic infrastructure used on the robot, and 
Section V reports our initial results of experimental tests on the robot. Section VI concludes the work.

\section{DESIGN CONCEPT}

The basic design concept of this leg-wheel hybrid platform is to include a "transformation mechanism" which is capable of deforming a specific portion of the body to act as a wheel or as a leg. From a geometrical point of view, a wheel usually has a circular rim and a rotational axis located at the center of the rim. The rim contacts the ground and the rotational axis connects to the moving platform at a point referred as a "hip" joint shown in Figure 2(a). On the flat ground the contact point is directly below the hip joint with a fixed distance (i.e. radius of the circular rim), thereby fixing height of the hip related to the ground. In contrary, legged platform in general doesn't have any confined geometrical relation between the hip and the contact point; thereby their relative position varies frequently during locomotion. Thus, shifting the hip point out of the center of the rim implies the locomotion switching from the wheel mode to the leg mode. This motivates us to design a mechanism which can directly control the relative position of the hip with respect to the rim. Since a circle is a 2-dimensional object, the straight forward method is to move the hip point along the radial direction of the circle (i.e. the rim).

The inertia of the leg and the geometrical space taken by the leg strongly affect the functionality and agility of legged motion. To decrease the inertia of the moving rim, the rim is supported by a single spoke, which also acts as the housing of the moving hip point. To reduce the space taken by the rim, in the leg mode the rim is folded in half (hereafter referred as a half-circle leg, similar to the legs on the second generation of RHex). With active actuation on the hip rotation and on the radial motion of the hip, the leg itself is equivalently to have 2 active degree of freedom (DOF) along with two principal axes in the polar coordinate in the sagittal plane depicted in Figure 3(a). Comparing to RHex [13] with only 1 active DOF per leg, though the structure of the proposed system is more complicate, it also greatly increases the freedom to adjust the configuration and contact timing of legs, thereby providing the opportunity to explore different behavioral subspace.

The general wheel platform has four wheels; thus, we adopt this methodology directly in the robot design. In the wheel mode shown in Figure 2(b), the locomotion behavior of this platform is similar to that of normal 4wheel-drive vehicles. The distances between the hip joints and the rims are fixed at a value equal to the radius of the rim, and the rotation motions at the hip joints are active to drive the platform forward or backward. Since the radial DOF rotates but not lies vertically toward ground, this active DOF can't be utilized to adjust the height of the robot as the active suspension. The front wheels can be steered to achieve turning motion according to (a)

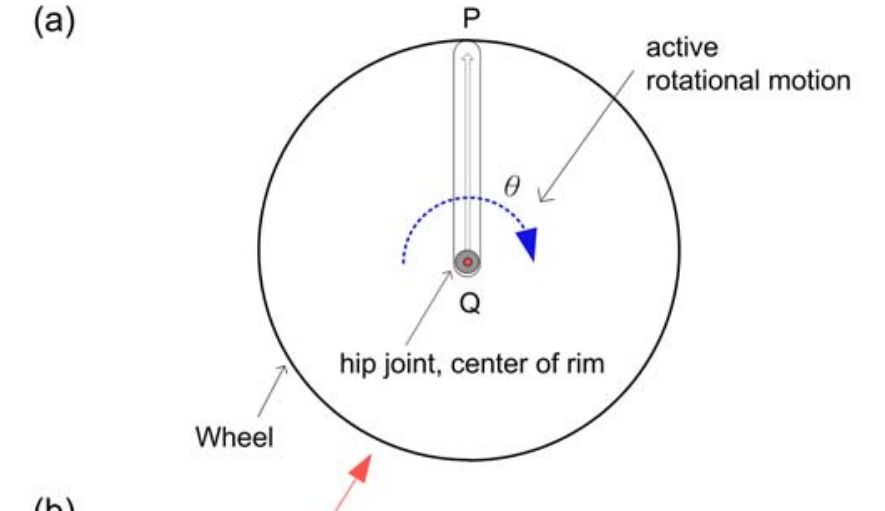

(b)

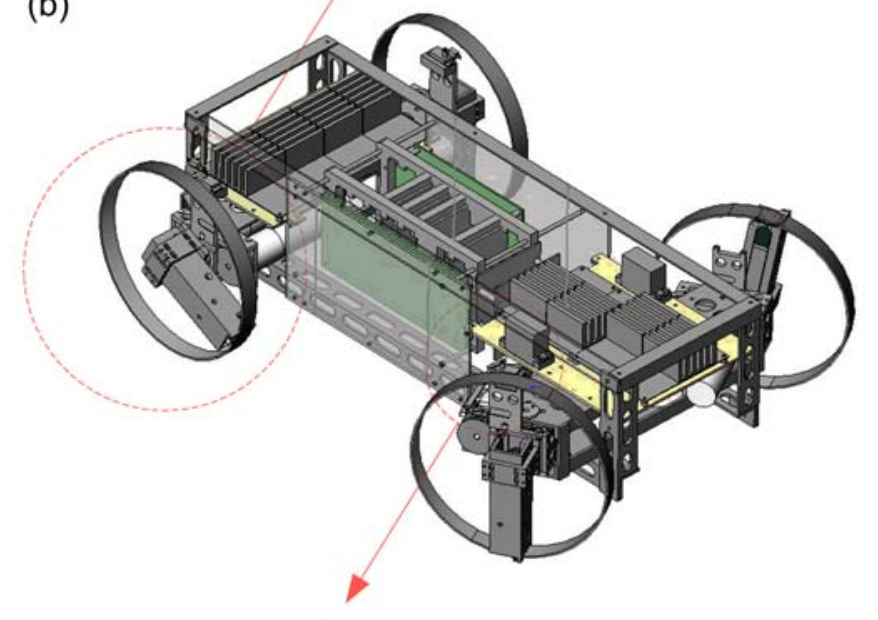

(c)

(d)
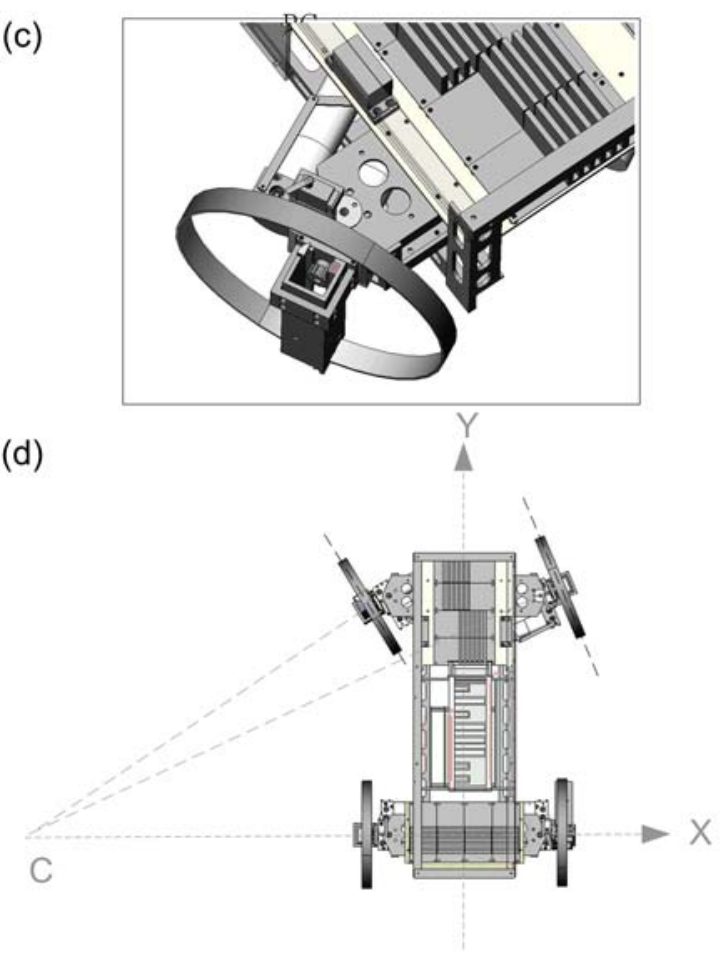

Fig. 2. Wheel mode: (a) detailed geometric illustration of the wheel; (b) CAD drawing of the robot operated in the wheel mode; (c) blow-up of the steering mechanism; (d) Ackermann steering geometry. 

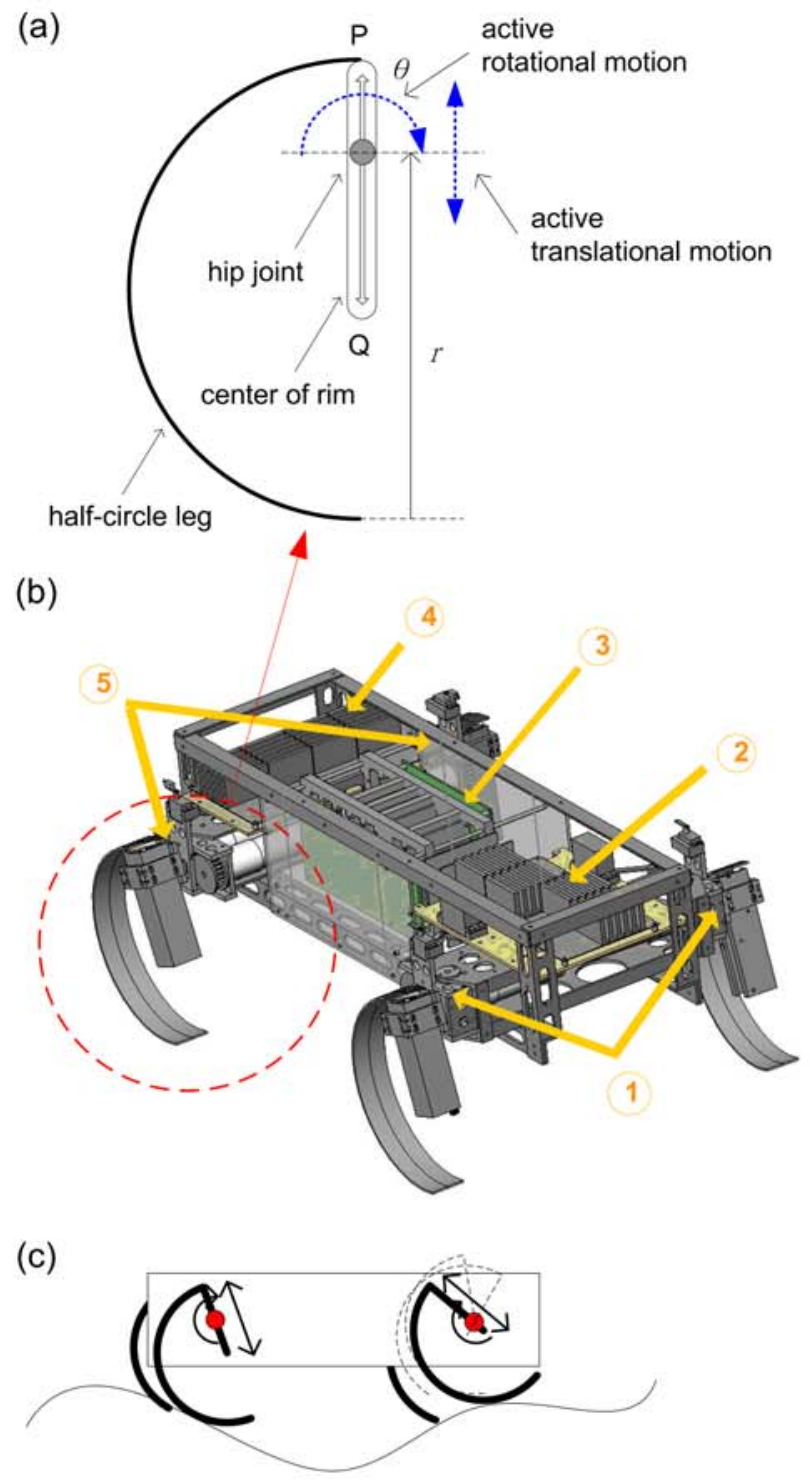

(d)

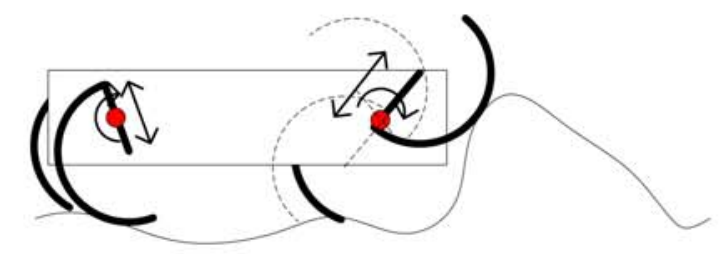

(e)

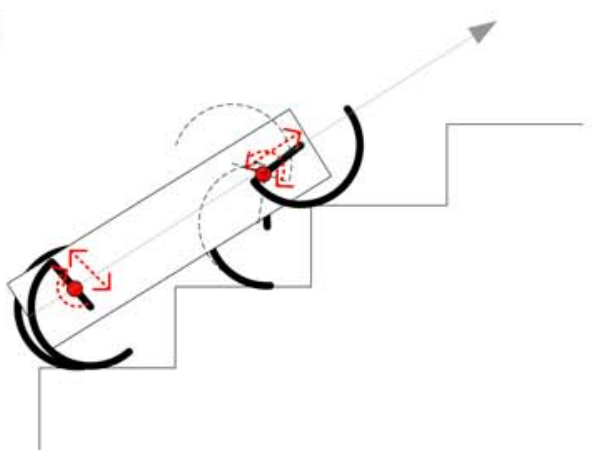

Fig. 3. Leg mode: (a)detailed geometric illustration of the leg; (b) CAD drawing of the robot operated in the leg mode; scenarios of leg locomotion: (c) walking on the rough terrain, (d) climbing $\mathbf{4 6 8 4}$ cross obstacles, (e) stair ascent
Ackermann steering geometry [18] shown in Figure 2(d), where the instant centers of all four wheels coincide in a single point $C$ (i.e. center of turning) on the extended line of real hip axis.

In the leg mode where the rim is folded in half and the hip point is moved close to the rim, the platform is transformed into a quadruped robot shown in Figure $3(\mathrm{~b})$. The change of leg length is achieved by changing the position of the hip joint in the spoke, and swing of the leg is driven by the active rotational joint at the hip. The leg mode is designed for the platform to have the capability to cross various uneven terrains. For example, (1) on the rough terrain where the behavior of the robot operated in the wheel mode is too bumpy, the robot operated in the leg mode with swing and extract/retract motion shown in Figure 3(c) may cross the terrain more smoothly; (2) on the terrain with large obstacles, the swing motion of the leg is replaced by the full rotating motion shown in Figure 3(d) so the robot can climb across the obstacles; (3) four 2-DOF legs can also be coordinated so the platform can perform stair ascent or descent shown in Figure 3(e), which is currently under investigation.

\section{LEG-WHEEL TRANSFORMATION MECHANISM}

The switching between the wheel mode and the leg mode of this leg-wheel hybrid robot is achieved by the transformation mechanism which includes a 2-DOF driving mechanism detailed in Section III-A and a legwheel switching mechanism detailed in Section III-B.

\section{A. Construction of the 2-DOF Driving Mechanism}

The 2-DOF driving mechanism includes two active driving motions on the leg-wheel component: rotation of the spoke and translational adjustment of "active spoke" defined by the distance between the hip joint and the rim. Equivalently, if the hip joint is defined as the origin, the 2-DOF mechanism is indeed driving the rotation $\theta$ and the translation $r$ in polar coordinate.

The rotational DOF of the spoke, equivalent to $\theta$, is driven by the rotational motion of the square sleeve, which is further driven by motor 1 through a belt transmission system composed by two pulleys and a timing belt (no speed reduction). The rotation axis of the square sleeve is the axis of the hip joint. A CAD model of this portion is illustrated in Figure 4(a).

The length change of "active spoke", the change of translational DOF $r$, is achieved by the sliding motion between the spoke and the square sleeve, and in between there are 16 small roller bearings installed to provide smooth sliding motion with low clearance. A rack installed on the spoke is driven by a pinion which connects to motor 2 directly.

As the result, the kinematic mapping between the inputs (i.e. motor rotations) and the outputs (i.e. leg motion) can be formulated as 

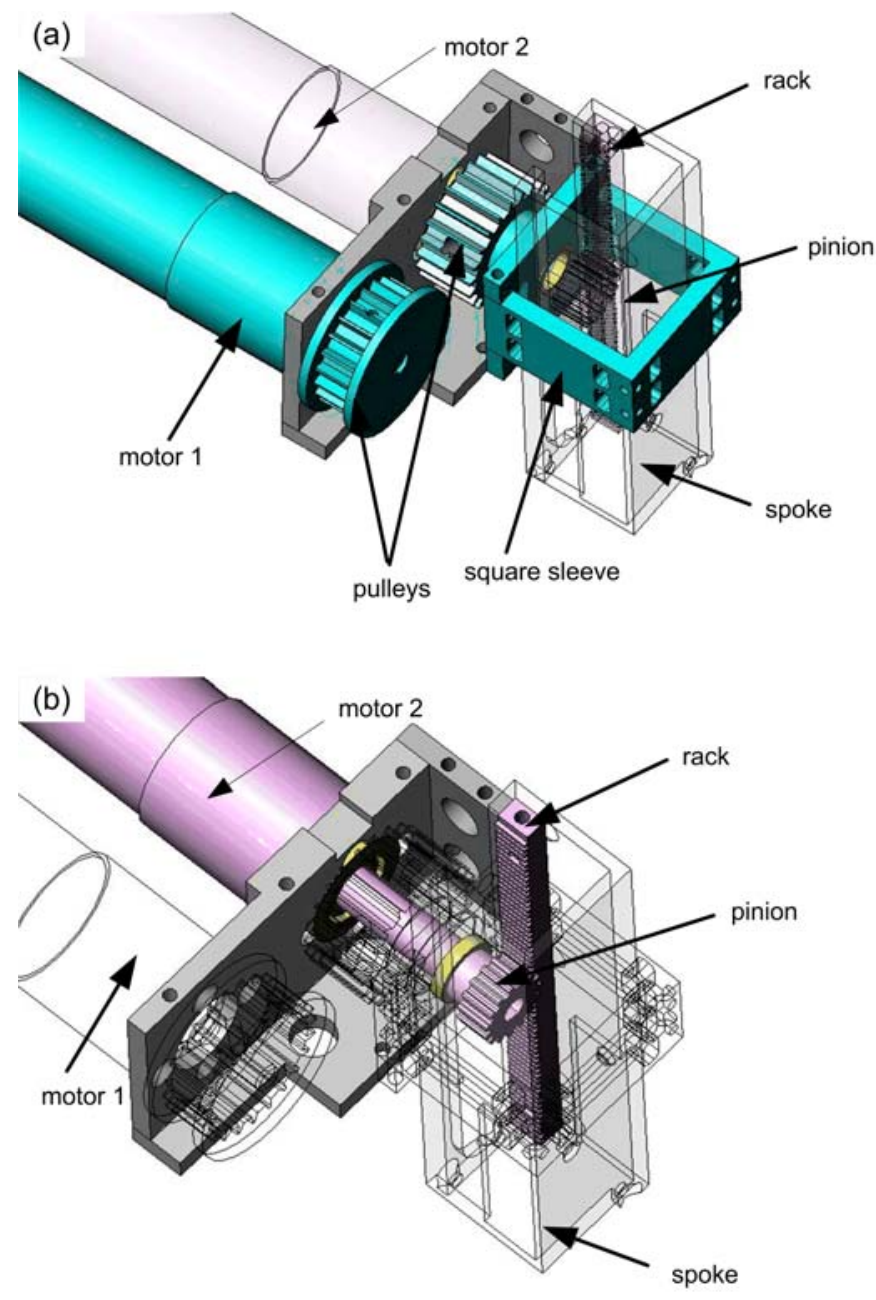

Fig. 4. 2-DOF driving mechanism: (a) rotational DOF; (b) translation DOF.

$$
\left[\begin{array}{l}
\dot{r} \\
\dot{\theta}
\end{array}\right]=\left[\begin{array}{cc}
a & -a \\
1 & 0
\end{array}\right]\left[\begin{array}{c}
\dot{\phi}_{1} \\
\dot{\phi}_{2}
\end{array}\right]
$$

where $\dot{\phi}_{1}$ and $\dot{\phi}_{2}$ are the rotational speed of the motor 1 and 2, respectively, and $a$ is the radius of the pinion which is the transformation factor from the rotational motion to the translational one.

When the robot is in the wheel mode, the rim must rotate with respect to the hip joint with a constant distance equal to radius of the rim, and this set point position control requires rotational motion of both motors with equal speed during locomotion. In the leg mode, both $\dot{r}$ and $\dot{\theta}$ change frequently so both motors are in motion. Thus, this design avoids using the motors in the stall mode during locomotion, which reduces the problems of current overload and heat generation in motors. In addition, both motors installed on the body reduces weight and inertia of the leg, which improves dynamic and control characteristics of the leg.

\section{B. Leg-wheel Switching Mechanism}

The switching between one full-circle rim (wheel mode) to two half-circle rims (leg mode) is driven by a micro RC servo motor installed inside the spoke shown in Figure 5. One half-circle rim is mounted in the spoke directly, and the other one is mounted on the rotating horn on top of the RC servo. The $180^{\circ}$ rotational motion of the $\mathrm{RC}$ servo swings the half-circle rim next to the other half-circle rim (leg mode) or away from the latter (wheel mode). In certain modes the spoke rotates continuously with respect to the hip joint; as the result, power and signal lines can not be hardwired from the robot body to the micro RC servo directly unless a slip ring is installed in between. Since the arrangement of the transmission mechanism around the hip area is already complicated, the installation of a slip ring is not feasible. Instead, a connector controlled by a mini RC servo motor shown in Figure 5 is installed. A male connector installed on top of the mini RC connects to the power and signal lines to the body, and a female connector installed on the spoke connects the lines to the micro $\mathrm{RC}$ which is in charge of the swing of the half-circle rim. The connectors are not connected to each other unless the leg-wheel switching operated is proceeded.
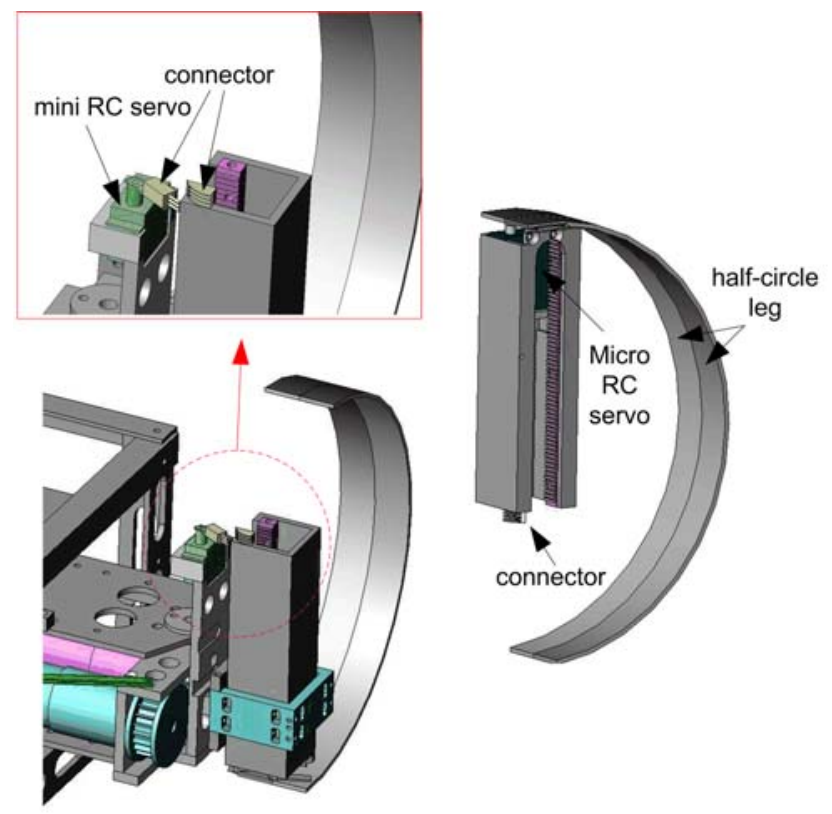

Fig. 5. Leg-wheel switching mechanism. The configuration of the leg-wheel component is controlled by a micro RC servo which swings one half-circle leg to let it away from the other one (wheel mode) or next to it (leg mode). The connectivity of power and signals from the robot body to the micro RC serve is controlled by a mini RC servo.

\section{Mechatronics}

The main computation power on the robot is a $400 \mathrm{MHz}$ real-time embedded control system (cRIO-9014, National Instruments) operating at $1 \mathrm{KHz}$ loop rate, together with a $3 \mathrm{M}$ gates field-programmable gate arrays 
(FPGA) embedded chassis (cRIO-9104) set to run at $10 \mathrm{kHz}$. The later one directly connects to analog $\mathrm{I} / \mathrm{O}$ (NI 9205 and NI 9264) and digital I/O (NI 9401 and NI 9403) modules which further connect to various sensors and actuators on the robot.

Software infrastructure includes three portions: PC, RT, and FPGA. A laptop PC is the remote control center which communicates with real-time processor (RT) on the robot via $802.11 \mathrm{~b}$ wireless standard. PC controlled by the operator only exchanges essential information with the RT, such as high level commands to drive robot in different modes, crucial motor and electronic status for health monitoring, and data logging. Most computation are executed in the onboard RT processor, and some algorithm requiring high loop speed are compiled in the FPGA, such as PID control of DC motors, encoder reading, and $\mathrm{RC}$ servo commanding.

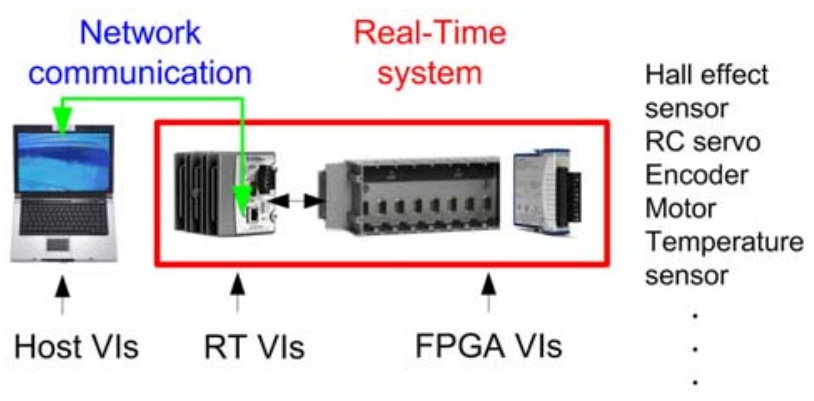

Fig. 6. Mechatronic infrastructure

The relation of different modes the robot can operate is shown in the Figure 7. After the robot is powered on, calibration of leg configuration is required, which finds the absolute geometric relations of two active DOFs on each leg/wheel with respect to the robot, or technically speaking, to define the absolute zero of encoder readings. The configuration is calibrated by the relative position between a hall effect sensor and a magnet with known positions. The calibrated robot can be operated either in the leg mode or in the wheel mode, depending on the rim configurations (i.e. wheel or half-circle leg). The leg-wheel switching can be performed after calibration mode or in the sit mode. Wheel mode includes standing, driving, and sitting three different actions. Leg mode for now includes standing, walking, and sitting. In the future various other motion modes will be explored, such as jogging, running, stair ascent/descent, and etc.

Various sensors are installed for status monitoring, including temperature sensor on the motors and power amplifier chips to prevent overheat problems, voltage and current measurement sensors to track the status of power consumed from battery, and various LEDs to indicate status of hall effect sensors and the loop operation.

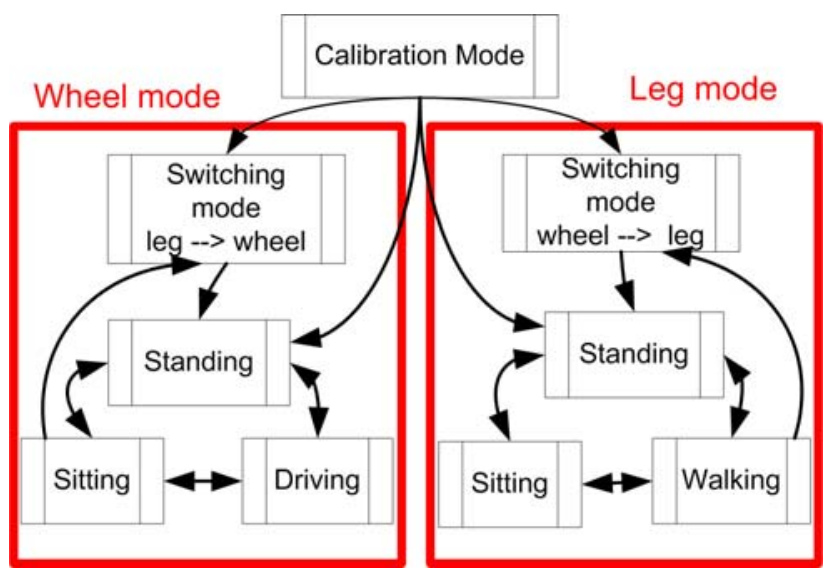

Fig. 7. Behavioral modes of the robot

TABLE I

Specifications of Quattroped

\begin{tabular}{|l|l|}
\hline $\begin{array}{l}\text { Length } \\
\text { body } \\
\text { leg-to-leg }\end{array}$ & $600 \mathrm{~mm}$ \\
\hline Width & $450 \mathrm{~mm}$ \\
body & $190 \mathrm{~mm}$ \\
Leg-to-leg & $410 \mathrm{~mm}$ \\
\hline $\begin{array}{l}\text { Height } \\
\text { body } \\
\text { standing (leg mode) } \\
\text { standing (wheel mode) }\end{array}$ & $140 \mathrm{~mm}$ \\
\hline Ground clearance (leg mode) & $190 \mathrm{~mm}$ \\
\hline Wheel diameter & $160 \mathrm{~mm}$ \\
\hline Weight & $216 \mathrm{~mm}$ \\
body & $8680 \mathrm{~g}$ \\
leg (each) & $380 \mathrm{~g}$ \\
total & $10500 \mathrm{~g}$ \\
\hline Driving actuators & \\
DC motors (8) & Maxon RE30 \\
High-torque RC servo (2) & Futaba 3305 \\
\hline Leg-wheel switching actuators & Hitec HS-85MG \\
Micro RC servo (4) & Futaba 3104 \\
Mini RC servo (4) &
\end{tabular}

\section{EXPERIMENT RESULTS}

As shown in Figure 3(b), there are six main blocks of components on the robot: (1) front legs, each with 2 DC driving motors, a transformation mechanism, and a four-bar linkage for steering; (2) front motor amplifiers, settled in the upper-front side of the robot; (3) computation center installed at the center of the robot, including real-time operating system, FPGA, I/O, and power management and distribution system; (4) back power amplifier, settled in the upper-back side of the robot; (5) rear legs, each with two DC driving motors and a transformation mechanism. Specifications of the robot are listed in Table I.

Figure 8 shows the sequence images of the robot operated in different modes, which are extracted from the associated movie along with this paper submission. The first row shows the wheel-to-leg switching, followed by the second row showing the robot stand-up in the leg 


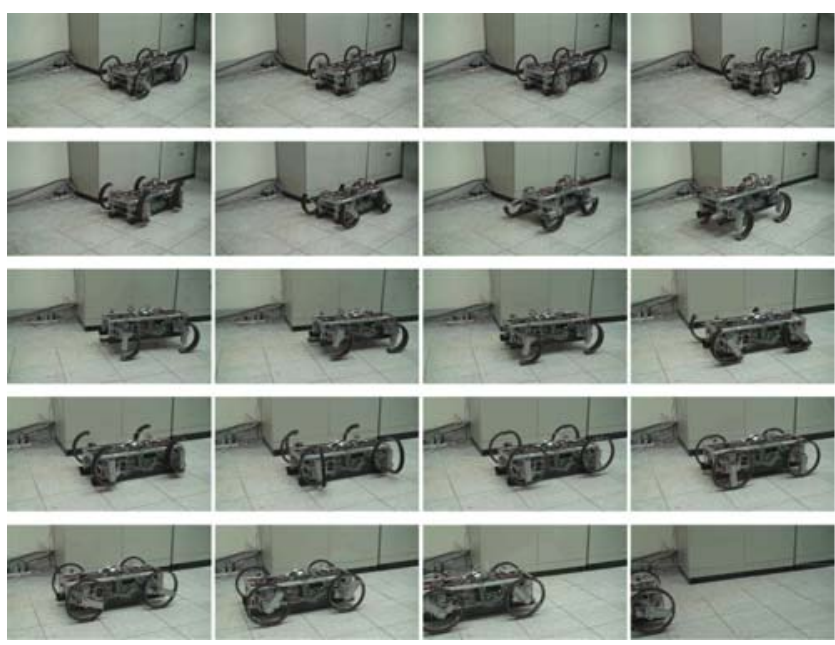

Fig. 8. Images of the robot operating in different modes - Top row: wheel-to-leg switching; 2nd row: stand-up (leg mode); 3rd row: walking and sit-down; 4th row: leg-to-wheel switching; bottom row: stand-up (wheel mode) and driving.

mode. The third row showing robot walking in crossover gait, and then sit-down. Similarly, the forth and fifth rows show the leg-to-wheel switching, stand-up in wheel mode, and then driving.

The video shows the robot operating in several different modes: starting with the calibration of leg configuration, followed by wheel-to-leg switching, robot standup in the leg mode, walking in the crossover gait, and then sit-down. The second portion includes leg-to-wheel switching, stand-up in the wheel mode, and then driving.

\section{CONCLUSIONS AND FUTURE WORKS}

We report on the design of a leg-wheel hybrid platform Quattroped which includes a transformation mechanism capable of directly changing the morphology of four wheels of the platform into 2 degree-of-freedom legs. The locomotion behaviors of this robot can be switched between a four-wheel-driven vehicle (wheel mode) and a quadruped (leg mode). We perform several experimental trails to test the validity of the design of the transformation mechanism and to evaluate the preliminary driving and walking behavior of the robot.

We are currently in the process of improving the robustness of the mechanical and mechatronic components of the robot, and in the meantime we are developing new legged behaviors, such as stair ascent/descent, jogging, running, and etc to improve its mobility on various different terrains.

\section{References}

[1] F. Michaud and S. Caron, "Multi-modal locomotion robotic platform using leg-track-wheel articulations," Autonomous Robots, pp. 137-156, 2005.

[2] G. Endo and S. Hirose, "Study on roller-walker," in IEEE International Conference on Robotics and Automation, vol. 1, 1999, pp. 2032-237.
[3] J. Hashimoto, T. Hosobata, Y. Sugahara, Y. Mikuriya, H. Sunazuka, M. Kawase, H. ok Lim, and A. Takanishi, "Realization by biped leg-wheeled robot of biped walking and wheel-driven locomotion," in IEEE International Conference on Robotics and Automation, vol. 1, 2005, pp. 2970-2975.

[4] J. Yuan and S. Hirose, "Research on leg-wheel hybrid stairclimbing robot,zero carrier," in IEEE International Conference on Robotics and Biomimetics, vol. 1, 2004, pp. 654-659.

[5] J. A. Smith, I. Sharf, and M. Trentini, "Bounding gait in a hybrid wheeled-leg robot," in IEEE/RSJ International Conference on Intelligent Robots and Systems, vol. 1, 2006, pp. $5750-5755$.

[6] H.Adachi, N.Koyachi, T.Arai, A.Skua, and Y.Nogami, "Mechanism and control of a leg-wheel hybrid mobile robot," in IEEE/RSJ International Conference on Intelligent Robots and Systems, 1999, pp. 1792-1797.

[7] C. Grand, F. Benamar, F. Plumet, and P. Bidaud, "Stability and traction optimization of a reconfigurable wheel-legged robot," The International Journal of Robotics Research, pp. 1041-1058, 2004

[8] S. Nakajima, E. Nakano, and T. Takahashi, "Motion control technique for practical use of a leg-wheel robot on unknown outdoor rough," in IEEE/RSJ International Conference on Intelligent Robots and Systems, vol. 1, 2004, pp. 1353-1358.

[9] M. Lacagnina, G. Muscato, and R. Sinatra, "Kinematics, dynamics and control of a hybrid robot wheeleg," Robotics and Autonomous Systems, pp. 161-180, 2003.

[10] V. Krovi and V. Kumar, "Modeling and control of a hybrid locomotion system," ASME Journal of Mechanical Design, vol. 121, no. 3, pp. 448-455, 1999.

[11] Y. Takita, N. Shimoi, and H. Date, "Development of a wheeled mobile robot "octal wheel" realized climbing up and down stairs," in IEEE/RSJ International Conference on Intelligent Robots and Systems, vol. 1, 2004, pp. 2440-2445.

[12] A. S. Boxerbaum, P. Werk, R. D. Quinn, and R. Vaidyanathan, "Design of an autonomous amphibious robot for surf zone operation: Part i mechanical design for multi-mode mobility," in IEEE/RSJ International Conference on Advanced Intelligent Mechatronics, vol. 1, 2005, pp. 1459-1464.

[13] U. Saranli, M. Buehler, and D. E. Koditschek, "Rhex - a simple and highly mobile hexapod robot," International Journal of Robotics Research, vol. 20, no. 7, pp. 616-631, 2001.

[14] M. Buehler, U. Saranli, and D. E. Koditschek, "Single actuator per leg robotic hexapod," United States Patent 6,481,513, 2002, mcGill University, The Regents of the University of Michigan, USA.

[15] S. HIROSE, T. SENSU, and S. AOKI, "The taqt carrier: A practical terrainadaptive quadru-track carrier robot," in IEEE/RSJ International Conference on Intelligent Robots and Systems, vol. 1, 1992, pp. 2068-2073.

[16] M. Guamieri, P. Debenesr, T.Inoh, E.Fukushima, and S. Hirose, "Development of helios vii: an arm-equipped tracked vehicle for search and rescue operations," in IEEE/RSJ International Conference on Intelligent Robots and Systems, 2004, pp. $39-45$.

[17] A. Masauaki, T. Takayama, and S. Hirose, "Development of "souryu-iii": Connected crawler vehicle for inspecbon inside narrow and winding spaces," in IEEE/RSJ International Conference on Intelligent Robots and Systems, 2004, pp. 5257.

[18] J. Y. Wong, Theory of Ground Vehicles. John Wiley and Sons Inc., 1993, p. 282. 